\title{
Innovations et stratégies logistiques dans la filière vitivinicole. Le rôle des proximités
}

Innovation and logistics strategies in the wine sector: the role of proximities

\section{Corinne Tanguy et Michel Martin}

\section{(2) OpenEdition}

1 Journals

\section{Édition électronique}

URL : http://journals.openedition.org/economierurale/4744

DOI : $10.4000 /$ economierurale. 4744

ISSN : 2105-2581

Éditeur

Société Française d'Économie Rurale (SFER)

\section{Édition imprimée}

Date de publication : 15 décembre 2015

Pagination : 83-99

ISSN : 0013-0559

\section{Référence électronique}

Corinne Tanguy et Michel Martin, « Innovations et stratégies logistiques dans la filière vitivinicole. Le rôle des proximités ", Économie rurale [En ligne], 349-350 | septembre-novembre 2015, mis en ligne le 15 décembre 2017, consulté le 15 novembre 2019. URL : http://journals.openedition.org/ economierurale/4744; DOI : 10.4000/economierurale.4744 


\title{
Innovations et stratégies logistiques dans la filière vitivinicole Le rôle des proximités
}

\author{
Corinne TANGUY, Michel MARTIN • CESAER, UMR 1041 INRA-AGROSUP, Dijon \\ corinne.tanguy@dijon.inra.fr, michel.martin@dijon.inra.fr
}

L'objectif de cet article est d'identifier comment les entreprises de la filière vitivinicole bourguignonne articulent les différentes formes de proximités pour gérer leur stratégie logistique aval (du départ de la cave au client) et les innovations qu'elles ont mises en place pour résoudre leurs problèmes logistiques. Une analyse réalisée, à partir des informations collectées auprès de 36 entreprises en Bourgogne, nous a permis de classer les entreprises en quatre catégories selon la combinaison de proximités élaborée : proximités géographique et organisée mais aussi proximité circulatoire qui tient compte de la qualité et de la fiabilité des transports.

MOTS-CLÉS : logistique, innovation organisationnelle, proximité circulatoire, filière vitivinicole

\section{Innovation and logistics strategies in the wine sector: the role of proximities}

The objective of this paper is to identify how companies of Burgundian wine sector articulate the proximities to manage their downstream logistics strategy (departure from the enterprise to client) and the innovations they have implemented to solve their logistics problems. An analysis, based on information collected from 36 companies in Burgundy, have allowed us to classify firms into four categories according to the articulation of proximities implemented by companies: geographical and organized proximities but also circulatory proximity that takes into account the quality and reliability of transport. (JEL: D22, L22, 031)

KEYWORDS: logistics, organizational innovation, circulatory proximity, wine sector

L a logistique est devenue un enjeu stratégique majeur dans de nombreux secteurs économiques, et notamment dans les industries agroalimentaires (Brulhart, 2002). En effet l'internationalisation croissante des marchés des vins entraîne une concurrence accrue, en particulier pour les vins d'entrée et de moyenne gamme, avec des normes environnementales de plus en plus strictes sur des marchés comme le Japon, le Canada ou le Royaume-Uni (Tanguy, Martin, 2015). En second lieu, sur le marché national, les entreprises de la filière sont confrontées à des changements des habitudes d'achat des clients, notamment ceux du circuit traditionnel des Cafés, Hôtels, Restaurants (CHR) (France Agrimer, 2014). Ces évolutions induisent des contraintes supplémentaires en matière d'organisation de la logistique amenant certains acteurs de la filière à innover et développer de nouvelles stratégies. Ces innovations peuvent être organisationnelles, en réorganisant en interne la chaîne d'informations et de produits, mais aussi en externe en développant les relations avec d'autres acteurs de la filière et/ou de la logistique. Les entreprises peuvent aussi innover technologiquement grâce aux systèmes et outils informatiques.

L'enjeu logistique est considérable pour le vignoble bourguignon puisque les exportations de vins représentent environ la moitié de la production totale, et que $38 \%$ de la production est expédiée en France, hors Bourgogne. La logistique facilite le partage et la coordination des flux informationnels et des biens entre tous 
les membres de la chaîne (Paché, Sauvage, 2004 ; Boissinot, Kacioui-Maurin, 2009). Or, mieux coordonner les flux de matières et de marchandises exige de faire adhérer les acteurs à des valeurs communes (Paché, 2006). L'établissement de liens de confiance et de proximité est alors un prérequis au partage de l'information entre les différents acteurs d'une chaîne logistique et des ingrédients primordiaux pour le bon fonctionnement de la chaîne logistique (El Ourdighi et al., 2008). La notion de proximité organisée (Rallet, Torre, 2004) développée par les tenants de l'école de la proximité reprend cette idée qu'au-delà de la proximité géographique, le transfert de connaissances et l'échange d'informations exigent une proximité en termes de codes de communication et de stabilisation d'un système de relations.

Dans cet article, nous étudions comment les acteurs de la filière vitivinicole bourguignonne gèrent leur logistique aval ${ }^{1}$ et les proximités qu'ils mobilisent pour se coordonner avec les autres entreprises de la filière, se mettre en relation avec les transporteurs ou logisticiens et accroître l'efficacité de leur logistique. Après avoir exposé les enjeux de la logistique et des innovations dans la filière vitivinicole, nous préciserons la méthodologie d'enquête utilisée. Puis, sur la base d'enquêtes, nous présenterons une typologie des stratégies logistiques des acteurs de la filière vitivinicole bourguignonne et discuterons ces résultats.

\section{Les innovations et choix stratégiques des entreprises en matière de logistique aval}

Notre analyse de la chaîne logistique se focalise sur les décisions stratégiques mises en œuvre par les entreprises vitivinicoles.

1. La logistique aval intègre les fonctions suivantes : l'embouteillage, le stockage, la préparation des commandes et le transport du vin de l'entreprise jusqu'aux clients.
Afin de parvenir à se coordonner avec des partenaires, les entreprises doivent dépasser leur projet individuel et élaborer des compromis organisationnels (Fulconis, Paché, 2011). En innovant en interne et en externe, l'objectif est d'accroître l'efficacité de leur logistique, de diminuer les coûts et d'améliorer la qualité du service (retards, erreurs, incidents), mais aussi d'anticiper le renforcement des normes environnementales (Masson, Petiot, 2013 ; Tanguy, Martin, 2015). Le rôle de l'accessibilité, de la vitesse et des délais permettant avec une fiabilité suffisante d'atteindre les marchés à desservir, est de ce point de vue fondamental pour évaluer la performance de la logistique (Masson, Petiot, 2013 ; Paché, 2006).

\section{Le rôle primordial de la coordination dans la logistique}

Depuis les années 1970, la logistique a connu de fortes évolutions quant à sa fonction et son rôle dans la chaîne de valeur (Porter, 1985). Dans les années 1970-1980, elle était limitée à une activité opérationnelle, visant à l'optimisation du transport, du stockage, de la production, etc. Elle était envisagée comme la gestion opérationnelle des flux physiques (Pimor, 2003 ; Colin, 2005), et le plus souvent en référence à la gestion du transport dans la chaîne d'approvisionnement (Michrafy et al., 2006). Elle est reconnue à partir des années 1990, comme « un véritable levier concurrentiel, permettant de piloter les flux transfonctionnels et transorganisationnels physiques, d'informations et financiers, dans les meilleures conditions de coût et de qualité de service » (GozéBardin, 2009). Elle se définit ainsi comme la « démarche de pilotage et de gestion des flux physiques de marchandises par des flux virtuels d'informations associées » (Colin, 2005). Dans un contexte de mondialisation des échanges, d'accélération des flux, d'hyperconcurrence et de renouvellement rapide des produits, la logistique 
constitue un puissant levier de compétitivité (Dornier, Fender, 2012). Faisant le lien entre l'offre et la demande, elle propose plusieurs leviers de croissance, de réduction des coûts et de mutualisation des ressources (Jouenne, Masion, 2007).

La logistique est donc aujourd'hui appréhendée comme une fonction stratégique dans l'entreprise. On ne parle plus de la Supply Chain ou chaîne d'approvisionnement, mais de la Supply Chain Management ou gestion de la chaîne d'approvisionnement (Pimor, 2003 ; Masson, Petiot, 2013). On cherche ainsi à intégrer les chaînes logistiques en partageant et coordonnant les flux d'information entre tous les membres, ce qui leur permet de mieux définir leurs rôles et responsabilités (Kempainen, Ari, 2003 ; Paché, Sauvage, 2004). De ce fait, la performance et la qualité logistique dépendent en tout premier lieu de la qualité de la coordination entre acteurs.

La logistique a ainsi été intégrée dans la filière lait depuis déjà une trentaine d'années et s'est étendue ensuite aux produits secs, puis aux animaux vivants. Elle est devenue dans ces secteurs un enjeu majeur au regard des nombreux défis et contraintes auxquels les entreprises font face aujourd'hui : flexibilité, réactivité, traçabilité, fragilité et durée de vie des produits, etc. (Brulhart, 2002). Pour autant, les entreprises recourent en majorité à l'externalisation de certaines activités comme celles d'entreposage, de préparation des commandes et de transport et avouent être rarement dans une logique de coopération logistique. L'embauche d'un responsable logistique est révélatrice par contre de l'importance stratégique accordée à cette fonction et elle se traduit par l'utilisation plus fréquente d'indicateurs de performance logistique (taux de service client, prévision des stocks, coûts logistiques, fiabilité des prévisions des ventes) (RivièreGiordano, 2012).
La filière vin est longtemps restée en France en marge des évolutions de la logistique (Réjalot, 2004). Les services logistiques offerts par les prestataires se limitaient, il y a encore peu de temps, uniquement à des services d'enlèvement, transport et livraison du vin. La crise des années 2000 et la concurrence des nouveaux pays producteurs ont commencé à remettre en question les pratiques courantes, y compris en matière de logistique. Le vin est un produit vivant et fragile et les variations brusques de température ou d'hygrométrie peuvent détériorer sa qualité, en particulier lors des exportations vers des destinations lointaines. Et contrairement aux autres secteurs, dans le cas de l'export, la logistique relève de la responsabilité de l'acheteur, ce qui entraîne une perte de contrôle du suivi du vin durant son transport et sa mise en marché. Par ailleurs, le fort accroissement des coûts du transport lié à la flambée du prix de l'énergie ainsi que l'émergence de la problématique du développement durable (Tanguy, Martin, 2015) renforcent aujourd'hui la nécessité pour les entreprises d'intégrer la logistique dans leur stratégie globale.

Les entreprises du secteur réalisent donc de plus en plus l'importance de la gestion de la chaîne logistique et l'impact positif de sa performance (Adamo, 2004). Les notions d'accessibilité, et donc de vitesse et délais auxquels seront livrés les clients, ainsi que de fiabilité de service, sont primordiales dans les circuits logistiques (Burmeister, Colletis-Wahl, 1997 ; Paché, 2006 ; Masson, Petiot, 2013). Parallèlement, les prestataires logistiques intervenant dans le secteur vitivinicole ont élargi leur gamme de services et offrent aujourd'hui des services complémentaires : stockage, préparation des colis, habillage, conditionnement, etc. (Réjalot, 2004).

En étant considérée comme une fonction stratégique, la logistique du vin peut favoriser les mécanismes de communication et 
de coopération, c'est-à-dire les interfaces (Poirel, Bonet, 2007). Elle constitue un moyen pour les acteurs d'améliorer les performances individuelles et collectives, mais aussi la qualité de leur coordination avec des partenaires (Adamo, 2004 ; Michrafy et al., 2006).

\section{Logistique et combinaison des proximités entre acteurs}

La coordination des flux de matières et de marchandises exige l'adhésion des acteurs à des valeurs communes (Paché, 2006). Une proximité en matière de codes communs de communication et l'établissement d'une certaine confiance sont alors des ingrédients primordiaux pour le bon fonctionnement de la chaîne logistique. Ce sont en effet des prérequis au partage de l'information entre les différents acteurs d'une chaîne logistique (El Ourdighi et al, 2008). L'économie de la proximité est justement née de la préoccupation commune de chercheurs autour de l'analyse de la coordination entre les acteurs (Bouba-Olga et al., 2008), en postulant d'emblée le fait que la coordination repose sur l'articulation de deux formes de proximité, la proximité géographique et la proximité organisée.

La proximité géographique ${ }^{2}$ indique si les acteurs d'une relation sont proches ou éloignés, et elle peut faciliter l'établissement de normes partagées entre ces acteurs (Rallet, Torre, 2004 ; May, 1999 ; Gilly, Pecqueur, 2000). Cependant, si la proximité géographique favorise les interactions, le fait d'être localisés à proximité ne suffit pas pour engendrer une action coordonnée des acteurs avec leurs voisins proches (Markusen, 2000 ; Boschma, 2004 ; Rallet, Torre, 2004 ; Shearmur, 2011 ; Tremblay et al., 2003 ; Filippi, Torre 2003; Zimmermann, 2008). Celles-ci

2. Elle se calcule en prenant en compte la distance kilométrique qui sépare les acteurs en termes de temps et/ou le coût de transport. naissent principalement dans le contexte d'activités organisées. Ainsi, des acteurs proches géographiquement peuvent coopérer plutôt avec des partenaires éloignés géographiquement s'ils ont développé ensemble une proximité organisée. Celleci rend compte du fait que les partenaires de la coopération vont partager des règles communes qui leur permettent de se coordonner. (Torre, Rallet, 2005 ; Bouba-Olga et al., 2008 ; Bellet et al., 1998). Selon Boschma (2004), cette forme de proximité peut varier en intensité. Ainsi, elle sera plus forte si les acteurs se trouvent à l'intérieur d'une entreprise ou d'un réseau organisé hiérarchiquement comme un groupe d'entreprises, que dans le cadre d'un partenariat entre entreprises indépendantes.

De manière à analyser les coordinations et les flux de marchandises entre les entreprises des secteurs industriels, du transport et de la logistique, certains auteurs proposent de prendre en compte également la notion de proximité circulatoire. Celle-ci concerne la capacité des entreprises à maîtriser et à contrôler des échanges coordonnés de biens (Frigant, 1996 ; Masson, Petiot, 2013). Elle prend en compte d'autres dimensions que la seule accessibilité temporelle (distance-temps) proposée dans la proximité géographique, comme les fréquences, la structure des coûts, la qualité et la fiabilité des transports (retards, erreurs, incidents) (Burmeister, Colletis-Wahl, 1997). Ainsi, outre la qualité et la fiabilité des transports, la proximité circulatoire dépend aussi de la qualité des infrastructures et de la proximité géographique des plates-formes, et des nœuds de communication (Paché, 2006). La proximité circulatoire sera élevée si la prestation des transporteurs et les infrastructures sont de qualité et si les nœuds de communication sont proches ${ }^{3}$.

3. En l'occurrence, les vignobles bourguignons sont situés le long d'axes de circulation et proches de Paris. 
La proximité circulatoire intègre donc des dimensions spatiales (distance, temps) et des dimensions non spatiales : fiabilité, flexibilité, traçabilité, coût, adaptation aux contraintes... (Burmeister, 2000). Les dimensions non spatiales vont à de nombreux égards dépendre du type de relations instaurées entre l'entreprise et ses partenaires, et donc de la proximité organisée qui permet la mise en œuvre de procédures de gestion communes et qui facilite la coordination des activités interdépendantes (Frigant, 1996 ; Masson, Petiot, 2013).

L'association des proximités organisée et circulatoire permet de gommer en partie la distance avec les clients (Frigant, 1996) et elle tend à relativiser le rôle de la proximité géographique sans toutefois le supprimer, en particulier dans la phase de mise en place de la relation (Burmeister, Djellal, 2004). Finalement, la proximité géographique est une ressource latente, qui ne peut être activée que par la proximité organisée, et qui permet de faciliter les coopérations entre les acteurs en matière de logistique. Ainsi, l'externalisation totale de la logistique d'une entreprise nécessitera une forte proximité organisée, facilitée d'un point de vue opérationnel par la mise en œuvre d'une proximité circulatoire (Frigant, 1996 ; Masson, Petiot, 2013), proximité qui sera favorisée à son tour par une proximité géographique avec le prestataire logistique. En réalité, selon la position dans la Supply Chain et le mode de pilotage des flux, le besoin de proximité géographique des acteurs sera plus ou moins important (Masson, Petiot, 2013 ; Paché, 2006). En définitive, on peut s'attendre à des combinatoires de proximité singulières qui vont dépendre de la localisation des cocontractants, du besoin plus ou moins important de proximité géographique entre les acteurs, du besoin d'échanger des informations et enfin de la nature de la relation entre cocontractants. Le rôle de la proximité organisée est ainsi primordial, que ce soit entre les membres d'un groupe, d'une Union de coopératives ou lors de la mise en place d'un apprentissage avec d'autres entreprises pour mutualiser ou externaliser la logistique (Masson, Petiot, 2013). L'instauration d'un climat de confiance avec les importateurs et d'une proximité organisée est particulièrement importante en cas d'export, lorsqu'il s'agit de faire parvenir le vin aux clients avec toutes les conditions requises de maîtrise de la qualité du produit.

\section{Quelles innovations en matière logistique dans la filière vitivinicole?}

Un secteur traditionnel comme celui du vin n'est pas nécessairement de «basse technologie » et de faible intensité de connaissances : il peut être innovant au même titre que d'autres secteurs industriels (Reboud et al., 2014). Face au modèle de «terroir» des pays européens, un certain nombre de facteurs ont permis, depuis les années 1990-2000, à un nouveau modèle de se développer dans les pays souvent qualifiés de « nouveaux producteurs ». L'évolution des habitudes de consommation de vins, et en particulier la baisse des volumes consommés dans les pays traditionnellement producteurs, l'importance croissante des aspects marketing et la progression des ventes dans les circuits type grande surface $^{4}$, ont favorisé le rattrapage des pays émergents comme l'Australie ou la Californie. L'adéquation de l'offre des producteurs de ces pays avec les demandes du marché international (Giuliani et al., 2011) s'est opérée grâce à de nombreuses innovations. La puissance financière des groupes ou wineries et leurs capacités en termes de marketing et d'export, de management environnemental, de réduction des délais et

4. Ce circuit de distribution désavantage les petits producteurs dans la mesure où les grandes surfaces veulent acheter des quantités très importantes de vin et sont aussi «friandes » de labels ou autres signes « compréhensibles » pour les consommateurs. 
de certification ont également joué un rôle certain dans leur succès. Comme le notent Giuliani et al. (2011), l'innovation est le résultat de la $R \& D$ formelle, mais aussi de la mise en œuvre de nouvelles routines et de changements organisationnels.

La logistique permet d'assurer la liaison opérationnelle entre la production et la consommation (Masson, Petiot, 2013). Aujourd'hui, c'est la demande des consommateurs qui pilote en partie la production, en particulier sur le segment des vins d'entrée de gamme. Du fait de la forte réputation du vignoble bourguignon et $\mathrm{du}$ faible volume produit, ce dernier est relativement protégé, au moins pour les vins de moyenne et haut de gamme. En effet, la production ne parvient pas à satisfaire la demande nationale et internationale. Malgré tout, l'ensemble des entreprises de la filière sont confrontées à la nécessité de faire évoluer leur logistique, pour répondre aux évolutions déjà mentionnées (diminution des stocks, exigences en termes de réactivité et de délais, de fiabilité ou encore de prise en compte du développement durable). La réponse à ces exigences se traduit par de nombreuses innovations organisationnelles ${ }^{5}$ que des entreprises, y compris parmi les plus petites, mettent en place pour rendre plus efficace leur logistique. Ces innovations peuvent être internes à l'entreprise, comme c'est le cas de la création d'un poste de responsable logistique ou de procédures permettant de concentrer les envois. Ces innovations peuvent aussi être externes et concerner les relations avec les autres entreprises. Ainsi, la mutualisation de la logistique dans le cadre d'un groupe ou d'une Union de coopératives ou entre partenaires, ou bien encore l'externalisation totale de la logistique à un

5. Pour l'OCDE, les innovations organisationnelles regroupent les nouvelles méthodes dans les pratiques de l'entreprise, l'organisation du lieu de travail ou les relations extérieures avec des partenaires (Manuel d'Oslo, 2005). prestataire spécialisé, constituent bien des innovations organisationnelles. Ces différentes stratégies sont en effet des moyens pour les entreprises viticoles de se recentrer sur leur cœur de métier et de bénéficier de compétences complémentaires pour accroître leurs performances. Nous proposons à présent d'examiner les innovations organisationnelles, les stratégies logistiques et les coopérations mises en œuvre par les entreprises viticoles en Bourgogne, en focalisant particulièrement l'analyse sur les formes de proximité mobilisées par les acteurs.

\section{La méthodologie et les données}

Trente-six entreprises de la filière ainsi que six prestataires de services logistiques ont été enquêtés sur la base d'un questionnaire semi-directif. Des questionnaires adaptés à chaque acteur ont été élaborés. Ceux qui étaient destinés aux coopératives, viticulteurs et négociants comportaient des rubriques communes : 1) une présentation de l'entreprise et de ses activités, 2) des questions sur la logistique et les modes de commercialisation, 3) une partie sur les réseaux et modes de coopération, 4) une autre sur les innovations développées par les entreprises, 5) et enfin, des questions sur la prise en compte du développement durable, et des enjeux auxquels font face les acteurs de la filière vitivinicole en Bourgogne. Ces entretiens, d'une durée moyenne de $1 \mathrm{~h} 30$, se déroulaient en face à face avec les personnes en charge des questions logistiques : le responsable logistique ou plus généralement, le responsable de la structure. Un autre questionnaire a été construit pour les prestataires de services logistiques avec une architecture proche du précédent ${ }^{6}$.

6. Seul l'ajout d'une partie « services fournis aux acteurs de la filière » diffère du questionnaire précédent. 
Les entreprises ont été choisies selon un plan d'enquête qui prend en compte leur taille, le type d'entreprise (coopératives, négociants, viticulteurs) (encadré 1) et leur localisation dans les différentes petites régions viticoles bourguignonnes (Chablis, Côtes de Nuit, Côtes de Beaune, Côte Chalonnaise, Mâconnais). Par ailleurs, nous avons mené des entretiens auprès de cinq structures collectives qui gèrent pour les entreprises adhérentes l'intégralité ou une partie de la commercialisation et de la logistique de leurs vins.

\section{Encadré 1. La filière des vins de Bourgogne : chiffres clés 2011}

Le vignoble est structuré autour de 100 Appellations d'Origine Contrôlée. La filière se caractérise par une taille moyenne des exploitations en 2010 de 7,6 ha (à titre de comparaison, celle du vignoble bordelais est de 11,9 hectares). Le volume de production est faible : environ 1,5 million d'hectolitres, soit $3 \%$ de la production française, et les acteurs sont nombreux : 3800 viticulteurs, 250 négociants, 23 coopératives. La commercialisation des vins est assurée à $58 \%$ par le négoce, $26 \%$ par les viticulteurs, $16 \%$ par les coopératives.

Source : BIVB (Bureau interprofessionnel des vins de bourgogne), Centre de

Documentation

Le faible nombre d'entreprises enquêtées ne nous a pas donné la possibilité de mener une analyse de données statistiquement valide, et nous avons donc réalisé une analyse qualitative et construit une typologie. Nous avons en particulier utilisé le logiciel statistique Spad 7.4 qui permet d'analyser l'ensemble des données, y compris des variables textuelles. Une procédure permet d'éditer les mots et segments répétés en fonction de la fréquence ainsi que les phrases caractéristiques d'une classe d'individus.
La population étudiée est composée de 6 coopératives, 14 négociants et 16 viticulteurs. La taille moyenne des 36 entreprises est de 24 salariés et la médiane s'établit à 7. La taille moyenne des 6 structures collectives de gestion de la logistique, qui gèrent, partiellement ou totalement, la logistique des entreprises adhérentes, est de 12 salariés. Le nombre limité d'enquêtes, en particulier chez les petits viticulteurs, permet de dégager les tendances en cours sans pouvoir analyser finement les pratiques en matière de logistique. Par ailleurs, notre enquête n'appréhende que les relations directes que les entreprises établissent avec d'autres entreprises de la filière ou des logisticiens, sans avoir une vision d'ensemble de la logistique des vins, en particulier à l'export. Pour appréhender la chaîne globale, il aurait fallu mettre en place un autre dispositif méthodologique et enquêter notamment les importateurs.

Pour analyser nos données, nous avons constitué quatre classes selon la capacité des entreprises à combiner les proximités pour rendre plus efficace leur logistique. Notre objectif est de vérifier quelles sont les combinaisons de proximités mobilisées par les acteurs dans ce type de partenariats. Nous distinguons trois types de proximité qui sont par ailleurs fortement interconnectées entre elles ; la proximité géographique ${ }^{7}$, en référence à la distance et temps de transport entre acteurs ; la proximité circulatoire, qui rend compte de la qualité du transport des biens et la proximité organisée. Pour constituer les classes, nous avons utilisé des indicateurs (encadré 2) qui rendent compte de la combinaison des proximités entre acteurs (avec les prestataires logistiques et/ou les autres entreprises partenaires).

7. Nous considérons que la proximité géographique intègre la dimension spatiale de la proximité circulatoire. 


\section{Encadré 2. Les indicateurs des proximités mises en œuvre dans les coopérations}

Nous avons considéré que la proximité géographique entre acteurs est forte puisque les entreprises de logistique avec lesquelles les acteurs ont des relations sont toutes situées à moins de 35 kilomètres. Par ailleurs, les coopérations entre les acteurs de la filière se réalisent quasi exclusivement avec des partenaires situés dans l'une des cinq zones viticoles. De fait, la longueur moyenne d'une zone est de 55 kilomètres, ce qui se traduit par des distances comprises entre 30 et 80 kilomètres entre partenaires.

L'indicateur de proximité organisée est calculé en mobilisant les deux variables suivantes : - L'appartenance à un groupe ou à une Union de coopératives facilite l'élaboration d'une proximité organisée entre les entreprises et une capacité à gérer collectivement la logistique. Elle améliore la proximité circulatoire. En effet, la mise en réseau et la coordination des flux de produits et d'informations sont facilitées au sein d'une même organisation. D'autre part, elle permet de concentrer les envois, d'utiliser les services des transporteurs de fret et d'accroître ainsi les indicateurs de service (coûts, délais, etc.).

- La mutualisation de la logistique suppose de développer une proximité organisée entre les entreprises. Elle est le moyen, en particulier pour les entreprises de petite taille, d'accéder à des compétences non présentes dans l'entreprise, et ainsi de rationaliser la chaîne logistique en concentrant les envois. Elle permet, comme dans le cas d'un groupe ou d'une Union, de coordonner les flux d'informations et produits, et donc améliore la proximité circulatoire.

La proximité organisée est forte lorsqu'une des deux variables est positive.

L'indicateur de proximité circulatoire est évalué au regard de l'accessibilité en délais et en fonction de la qualité de services. Elle est forte lorsque le transport est réalisé par des affréteurs, faible lorsque l'entreprise utilise les services d'une entreprise de messagerie.

- Le recours aux transporteurs fret ${ }^{8}$, lot ou palette : Les prestataires de services logistiques collectent les envois des viticulteurs avec une qualité de services élevée (prix inférieurs, moins de casses, respect des délais, etc.). L'existence de ces services et le niveau de ces indicateurs révèlent alors une proximité circulatoire forte.

- Le recours aux transporteurs locaux de messagerie : une proximité géographique forte entre viticulteurs et transporteurs sera un élément majeur de la stratégie des transporteurs qui prennent en charge les envois en faible quantité des viticulteurs dans un rayon de 30 kilomètres. L'emploi de service de messagerie révèle une proximité circulatoire faible dans la mesure où les indicateurs de services (délais, qualité de services et coûts) sont inférieurs à ceux du fret.

\section{Les résultats}

Pour la grande majorité des entreprises de la filière des vins de Bourgogne, la perception de la logistique aval se réduit à la fonction transport. Les relations avec les transporteurs se font sur une base informelle et seule une grille tarifaire est définie pour une année ainsi que les services associés. Le choix des transporteurs se fait pour la totalité des entreprises sur des critères de qualité et de services, et dans un second temps de prix. Tous les acteurs notent que les vignobles bourguignons sont très proches de grands axes de circulation, de plates-formes logistiques et de logisticiens, ce qui facilite les expéditions de vins. Cette localisation garantit donc une bonne accessibilité et proximité circulatoire. Cependant, le vignoble s'étendant sur une bande de territoire de 300 kilomètres de long, il faut nuancer ce constat en fonction de la localisation de certains acteurs et des difficultés d'accessibilité qu'ils peuvent

\footnotetext{
8. Le fret ou palette ou lots concerne les envois en grande quantité, les colis sont palettisés, il n'y a pas de manutention puisque souvent le transporteur assure la livraison directe du client sans opération de groupage-dégroupage.
} 
Tableau 1. Les classes d'entreprises selon les indicateurs de proximité

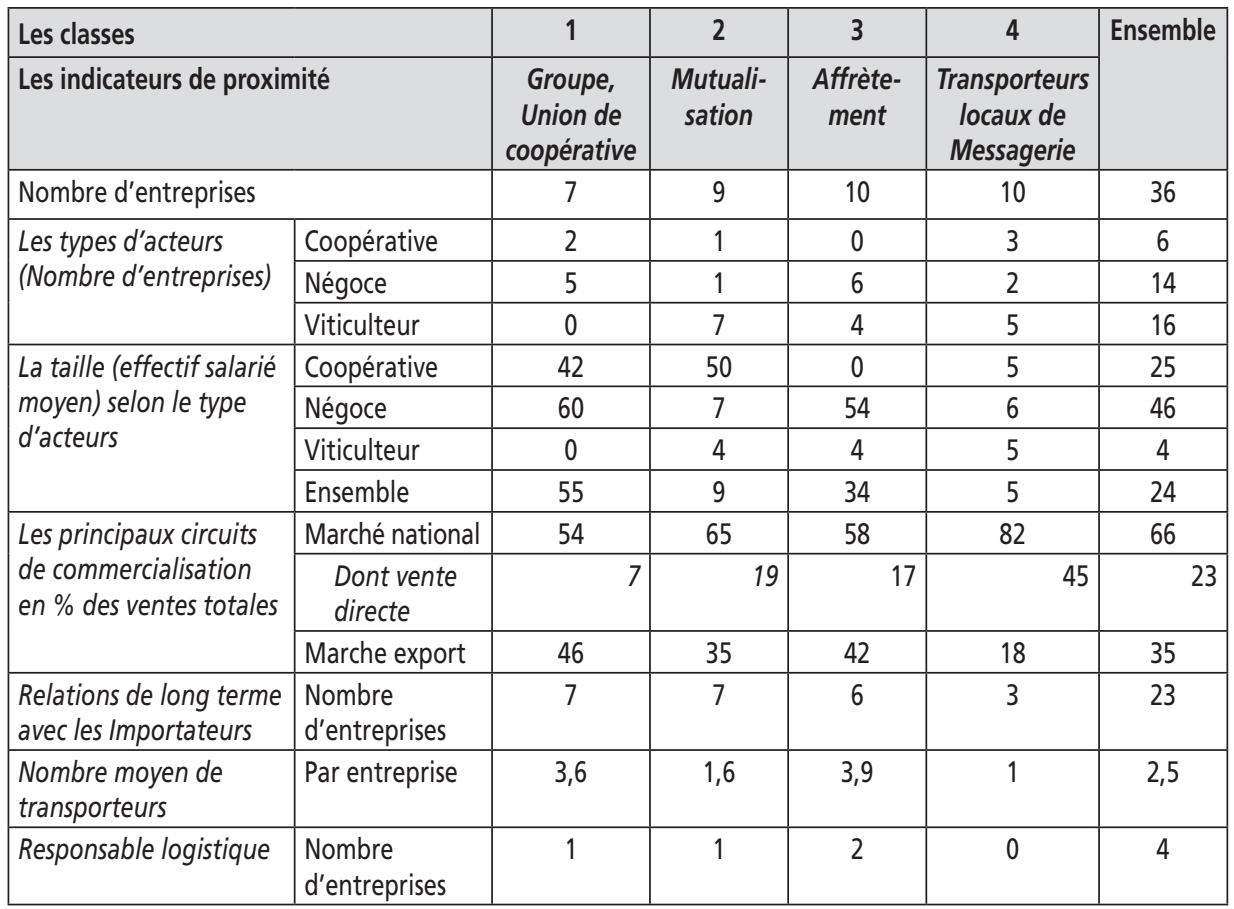

Source : les auteurs.

rencontrer. En raison de cet éloignement, et de manière à pouvoir coopérer, ces entreprises doivent arriver à construire une proximité organisée avec d'autres acteurs.

Dans le tableau 1, nous présentons la typologie en quatre classes obtenue en fonction de la combinaison des proximités développées par les acteurs. Des variables comme la taille, les circuits de commercialisation, les relations établies avec les importateurs, le nombre moyen de transporteurs avec lesquels travaille l'entreprise, ou la présence ou non d'un responsable logistique, permettent de décrire les classes obtenues.

Les acteurs de la filière viticole se répartissent en quatre classes en fonction de la combinaison des proximités et des stratégies logistiques qu'ils mettent en œuvre. Les classes 1 et 2 privilégient la combinaison des proximités organisée et circulatoire alors que les classes 3 et 4 mixent les proximités géographique et circulatoire. La proximité organisée joue également un rôle non négligeable dans l'établissement et la poursuite des relations de la classe 3 .

\section{Classe 1 - Des entreprises qui articulent} les proximités organisées et circulatoires au sein d'un groupe ou d'une Union de coopératives

Ces entreprises de grande taille (tableau 1) mutualisent leur logistique dans le cadre du groupe ou de l'Union de coopératives. Parmi elles, seule une entreprise a créé un poste de responsable logistique ${ }^{9}$.

9. Nous n'avons enquêté que les entreprises localisées en Bourgogne et non le niveau groupe. Nous ne savons donc pas si les groupes ont intégré ou non un poste de responsable logistique. Par contre, l'enquête auprès de l'Union des coopératives nous a permis de montrer le rôle stratégique du responsable logistique dans le cadre de l'Union et pour les coopératives adhérentes. 
Dans le cadre du groupe ou de l'Union, il y a souvent mutualisation et création d'une plate-forme unique pour l'ensemble des entreprises. Cette stratégie permet donc de concentrer l'essentiel des envois de vins pour travailler avec les transporteurs de fret et de négocier avec eux dans les meilleures conditions. Ces entreprises sont proches au niveau organisationnel, elles ont mis en place des procédures (y compris en termes d'outils informatiques) et des codes communs de communication et peuvent ainsi coopérer plus facilement.

Ainsi, certaines coopératives ont créé en Bourgogne une structure dédiée à la logistique : il s'agit de Blasons de Bourgogne qui s'avère être le projet collectif le plus abouti. Elle assure la commercialisation et l'ensemble de la logistique des vins des coopératives adhérentes pour les marchés export et la GMS. C'est le premier metteur en marché bourguignon avec $16 \%$ de la production viticole. Blasons de Bourgogne prend également en charge, grâce à une plate-forme commune, la logistique pour tous les autres circuits. Sa taille et son organisation permettent d'obtenir des coûts de transports très bas et des indicateurs de service très performants. Cette plate-forme située à Beaune se trouve près d'infrastructures autoroutières et des transporteurs et logisticiens ${ }^{10}$.

L'activité de ces entreprises à l'export est plus importante que celles des autres classes. Elles ont développé une organisation qui permet de garder un contrôle du transport malgré le choix du prestataire logistique à l'export par le client. Ainsi, toutes les entreprises ont des relations de long terme avec leurs importateurs et elles ont, grâce à ces relations, établi un climat de confiance mutuelle. Cette proximité organisée permet d'échanger des informations sur le choix de tel ou tel transporteur.

10. Ainsi se trouvent à Beaune de nombreuses sociétés de transport ainsi que le leader mondial en termes de transport du vin, la société Hillebrand, qui est implantée également à Bordeaux et Cognac.
Sur le marché national, ces entreprises travaillent avec des transporteurs « historiques » avec lesquels elles ont également des relations de long terme. Cependant, pour obtenir un haut niveau de service, elles mettent régulièrement en concurrence ces transporteurs avec d'autres entreprises. Elles ont mis en place un suivi mensuel des résultats des transporteurs et négocient les tarifs ${ }^{11}$ (prix moyen par bouteille inférieur à $1 €)$ en argumentant sur leur volume important de production ainsi que sur leur notoriété. Les transporteurs ont en effet intérêt à avoir des maisons prestigieuses comme clients. Il s'agit d'un argument commercial dans le milieu viticole bourguignon.

Par ailleurs, une entreprise a développé une forte proximité organisée avec un prestataire situé à quelques centaines de mètres pour externaliser totalement sa logistique aval et les fonctions suivantes : réception des commandes, stockage, préparation des commandes. Elle travaille avec lui depuis plus de vingt ans, ce qui lui a permis d'établir des liens de confiance et une réelle réactivité. Ce partenaire logistique modifie son organisation pour s'adapter à la saisonnalité des ventes de son client, en particulier lors de la période des foires aux vins. Les entreprises de cette classe combinent les proximités organisée et circulatoire.

\section{Classe 2 - Des entreprises qui mixent les proximités organisée et circulatoire pour coopérer}

Ces entreprises, dont la taille moyenne est de 9 salariés, sont majoritairement des viticulteurs. Une coopérative et un négociant appartiennent également à cette classe.

11. Les prix du transport dépendent non seulement de la stratégie organisationnelle de l'entreprise, mais aussi des volumes, de la distance à parcourir pour livrer le client et de la localisation de l'entreprise. En fonction de ces éléments, les tarifs pourront être plus ou moins intéressants. Il est à noter que le prix de la bouteille en lui-même n'influe pas sur le coût du transport. Le prix moyen mentionné est donc indicatif. 
Tableau 2. Les deux types de mutualisation du transport ou de la logistique

\begin{tabular}{|l|l|l|}
\cline { 2 - 3 } \multicolumn{1}{l|}{} & \multicolumn{1}{|c|}{ Structures de gestion de la logistique } & Regroupements pour gérer le transport \\
\hline Formes juridiques & $\begin{array}{l}\text { Société à responsabilité limitée (SARL) } \\
\text { ou Union de coopératives }\end{array}$ & Syndicat de vignerons \\
\hline Adhérents & Coopératives, viticulteurs & viticulteurs \\
\hline Objectifs & $\begin{array}{l}\text { Prendre en charge l'ensemble des } \\
\text { fonctions de la logistique aval }\end{array}$ & $\begin{array}{l}\text { Négociation tarifs transport et des } \\
\text { services associés }\end{array}$ \\
\hline Nombre de salariés & 20 & 0 \\
\hline $\begin{array}{l}\text { Fonctions } \\
\text { logistiques }\end{array}$ & $\begin{array}{l}\text { Commerciale, stockage, préparation des } \\
\text { commandes, facturation, suivi des clients, } \\
\text { transport }\end{array}$ & Transport \\
\hline Période de création & Entre 1980 et 1993 & $2006-2009$ \\
\hline
\end{tabular}

Source : les auteurs.

Ces entreprises adhèrent à des structures collectives auxquelles elles délèguent partiellement ou totalement leur logistique dans le cadre d'une relation partenariale. Ces relations se sont construites progressivement, permettant l'établissement de liens de confiance entre partenaires. Ceux-ci se sont traduits par la création ou l'évolution du projet commun et d'une proximité organisée de plus en plus forte entre les adhérents. Ces regroupements répondent à une logique commune, la possibilité en se regroupant de mieux négocier avec les transporteurs et ainsi réduire les coûts de transport. Ainsi, un viticulteur a vu sa facture de transport diminuer de $40 \%$ en adhérant à un groupement. Toutefois, il existe deux logiques organisationnelles (tableau 2) qui sous-tendent ces regroupements.

Dans le premier cas, la création d'une structure autonome émane de la volonté des entreprises. Elles ont intégré dès le départ dans leurs prérogatives la diminution des coûts du transport, mais aussi l'optimisation de la logistique en incluant partiellement ou totalement les fonctions comme le stockage, la préparation des commandes, la facturation, le suivi des clients. Par ailleurs, elles ont regroupé la production des adhérents pour constituer une gamme de vins plus large et diversifiée, afin d'intéresser d'autres clients et, en particulier, la GMS. Après une phase d'apprentissage, le projet collectif a pu évoluer pour inclure par exemple d'autres circuits de commercialisation comme l'export, grâce à l'embauche de commerciaux et la création de bureaux à l'international.

Dans le second cas, les regroupements associatifs ont été créés par des leaders ${ }^{12}$ ou acteurs moteurs de la filière (entretien $\mathrm{n}^{\circ}$ 42). Ici ce sont des viticulteurs, qui ont créé une dynamique permettant ainsi de fédérer d'autres viticulteurs proches géographiquement. L'organisation de chaque regroupement est le reflet du projet collectif des adhérents. Ainsi, comme dans le premier cas, les projets ont évolué progressivement en intégrant d'autres objectifs que les objectifs de départ. À titre d'exemple, un groupement a été constitué à l'origine pour obtenir de meilleurs tarifs lors des négociations avec les transporteurs de messagerie et des services supplémentaires.

12. Nous adaptons la notion de leader viticole développé par Jean-Marc Touzard (2008) selon lequel un «leader viticole » est défini par la reconnaissance d'un groupe de personnes dont il représente les intérêts, par son influence sur ce groupe, sans reprendre par contre la référence de l'auteur à la position stratégique du leader au sein des différentes organisations des marchés du vin. 
Aujourd'hui, il prend aussi en charge la négociation des tarifs avec les transporteurs de fret. Second exemple : deux coopératives, dont une localisée en Ardèche, ont créé une société indépendante pour assurer l'exportation de leurs vins en proposant une gamme plus large et bénéficier ainsi d'économies de variété (Jetin, Lung, 2000). Cette initiative permet de partager les frais du personnel, de diminuer les coûts et de réaliser des économies d'échelle.

Les entreprises en mutualisant leur logistique, ou bien en ayant un plus grand volume de production et une organisation intégrant la logistique, peuvent ainsi expédier la très grande majorité de leurs vins via l'affrètement. Elles font ainsi faire baisser significativement le coût du transport (prix moyen par bouteille supérieur ou égal à $1 €)$. Ces regroupements permettent aussi d'améliorer la proximité circulatoire en obtenant de meilleurs indicateurs de services en particulier en termes de qualité de service (délais, prix, casses, vols, etc.).

Cette organisation logistique collective offre ainsi l'avantage de constituer une gamme de vins plus conséquente et diversifiée, un volume de production suffisant

\section{Tableau 3. Les indicateurs de service ${ }^{13}$}

\begin{tabular}{|l|l|r|r|r|r|}
\cline { 2 - 6 } \multicolumn{1}{c|}{} & \multicolumn{1}{|c|}{ Classes } & \multicolumn{1}{c|}{1} & \multicolumn{1}{c|}{$\mathbf{2}$} & \multicolumn{1}{c|}{$\mathbf{3}$} & \multicolumn{1}{c|}{} \\
\hline Stratégie & Mutualisation & 3 & 11 & 1 & 1 \\
\hline \multirow{4}{*}{$\begin{array}{l}\text { Indicateurs } \\
\text { de service }\end{array}$} & Prix & 2 & 6 & 6 & 2 \\
\cline { 2 - 6 } & Qualité & 5 & 11 & 9 & 7 \\
\cline { 2 - 6 } & Service & 3 & 5 & 5 & 2 \\
\cline { 2 - 6 } & Qualité/prix & 0 & 2 & 2 & 2 \\
\cline { 2 - 6 } & Qualité/service & 3 & 5 & 5 & 2 \\
\hline
\end{tabular}

Source : les auteurs.

13. Le tableau 3 synthétise les réponses des acteurs à la question suivante : quels critères prenez-vous pour choisir un transporteur? Il permet de voir le nombre de mots répétés caractéristique d'une classe d'entreprises. À titre d'exemple, les entreprises de la classe 2 citent 11 fois le mot «mutualisation » et 6 fois le mot « prix ». pour vendre des vins dans le circuit GMS, et permet d'accroître les débouchés à l'export. À travers ces exemples de projets partenariaux, nous pouvons voir que les acteurs articulent les proximités organisée et circulatoire, mais aussi géographique dans la mesure où un des partenariats se développe entre acteurs situés dans le même village.

Classe 3 - Des entreprises qui combinent compétences internes et proximités circulatoire et géographique pour gérer leur relation avec les transporteurs

Cette classe est constituée par six négociants de grande taille parmi les plus réputés en Bourgogne et quatre viticulteurs. Une des spécificités de ces entreprises est qu'elles mobilisent surtout leurs compétences internes pour gérer la logistique.

Elles innovent en interne sur le plan organisationnel pour mettre en place une stratégie logistique efficace. Ces entreprises considèrent en effet que leur taille est suffisante et n'envisagent pas de coopérer. Elles ont mis en place une organisation de leur logistique et embauché un responsable logistique pour deux d'entre elles. Leur volume d'activité ainsi que leur capacité d'organisation leur permettent de consolider les expéditions pour mobiliser principalement des transporteurs affréteurs, stratégie qui se révèle la plus efficace sur le plan économique (Fassio, 2009). Les prix de transport sont inférieurs (prix moyen par bouteille de $1 €$ ) à ceux de la messagerie et les indicateurs de qualité de service supérieurs (tableau 3). De ce fait ces choix améliorent sensiblement la proximité circulatoire.

Ces entreprises travaillent avec plusieurs transporteurs (en moyenne 4) afin de couvrir l'ensemble de leurs besoins en fonction des quantités expédiées (fret ou messagerie) et des destinations. En effet selon un négociant les transporteurs sont plus ou moins efficaces en matière de prix et de service selon l'organisation de leur 
réseau. Il y a «des zones géographiques où ils sont plus compétitifs » (entretien $\left.\mathrm{n}^{\circ} 36\right)$. Certains seront par exemple spécialisés et très performants dans les livraisons à Paris et région parisienne.

Selon les entreprises, les relations avec les transporteurs sont stables même s'il n'y a pas de contrat formalisé. Chaque entreprise a des transporteurs " historiques » avec lesquels elle collabore depuis plusieurs années, voire plus d'une décennie. Les entreprises disent garder tout contrôle sur le choix des transporteurs, mais favorisent des partenaires fiables même si leurs prestations sont plus chères. Elles mobilisent surtout les transporteurs de fret sur la base d'une proximité géographique forte.

Les viticulteurs de cette classe disent s'organiser pour concentrer leurs envois pour travailler avec des transporteurs affréteurs pour diminuer les coûts et obtenir une meilleure qualité de service. Ainsi, une entreprise signale «qu'elle essaye d'optimiser les transports : regrouper les commandes, faire le maximum d'envois pour remplir les camions. Ainsi, la maison appelle des clients pour les informer que le camion va passer près de chez eux et voir s'ils ont besoin de produits dans le but de remplir au maximum les camions. La maison offre le transport à partir d'une palette $»\left(\right.$ entretien $\left.\mathrm{n}^{\circ} 18\right)$.

Finalement ces entreprises mobilisent surtout leurs ressources internes associées à une forte proximité circulatoire et géographique. L'activité export est importante et les relations avec leurs importateurs sont également majoritairement de long terme (6 sur 10), basées sur une forte proximité organisée.

\section{Classe 4-De très petites entreprises} qui s'appuient surtout sur la proximité géographique pour gérer leur relation avec les transporteurs locaux

Deux négociants, trois coopératives, cinq viticulteurs, tous de très petite taille déclarent n'avoir aucune stratégie logistique. Ces entreprises considèrent que leur métier s'arrête «à la porte de leur chai». Selon sept acteurs sur les dix, la logistique est secondaire et n'est pas intégrée dans leur organisation. Les trois autres s'organisent de façon informelle avec d'autres acteurs pour regrouper leurs envois ou possèdent des camions ou camionnettes pour livrer directement leurs clients cavistes ou restaurateurs et garder ainsi le contact avec eux.

$\mathrm{Du}$ fait de leur faible volume d'activité, ces entreprises travaillent exclusivement avec des transporteurs de messagerie locaux, spécialisés dans le ramassage auprès des domaines de vin en petites quantités. De fait, ce sont ces entreprises qui ont les performances logistiques les moins « bonnes », en termes de prix (prix moyen par bouteille supérieur à 1,50 €) et de taux de service. Les entretiens montrent que ces pratiques se traduisent par des taux de casse et de vol supérieurs à ceux des classes qui mobilisent les transporteurs de fret. Elles bénéficient de ce fait d'une faible proximité circulatoire.

Des relations de confiance ont été établies entre les vignerons et ces transporteurs, notamment grâce à la présence des mêmes chauffeurs depuis de nombreuses années. Ils ont réussi à instaurer un climat de confiance, à tel point qu'ils peuvent prendre directement les colis dans la cave du vigneron en cas d'absence. Ces transporteurs font preuve de beaucoup de souplesse en proposant des passages à des heures précises, l'enlèvement en petites quantités $^{14}$ auprès des viticulteurs. La proximité géographique a donc progressivement permis que se construise une

14. Le transport du vin par messagerie comprend trois phases principales, l'enlèvement ou ramassage, la traction, la livraison-distribution. Le coût total du transport se répartit en moyenne entre ces phases selon la proportion suivante : $20-25 \%, 35-40 \%, 35-40 \%$. 
proximité organisée. Les transporteurs concentrent sur leur plate-forme les colis qui seront ensuite expédiés grâce aux contrats passés sur de gros volumes avec d'autres transporteurs nationaux implantés en Bourgogne, "en exigeant d'eux une qualité de service que les petits viticulteurs ne peuvent pas obtenir seuls » (entretien $\operatorname{logisticien} \mathrm{n}^{\circ} 4$ ).

La stratégie de ces entreprises est axée vers le marché français et le développement du circuit de vente directe. Il représente déjà une part importante de leurs ventes. Par contre, leur activité export est faible.

Finalement, nous montrons que les entreprises enquêtées combinent les proximités pour gérer leurs partenariats. Les classes 1 et 2 articulent, elles, les proximités organisée et circulatoire. Cependant, l'établissement de liens de confiance entre les acteurs nécessite des rencontres fréquentes et une proximité géographique, permanente ou temporaire (Torre, 2008). La seconde classe mobilise ainsi la proximité géographique puisque nous trouvons dans cette classe un ensemble d'acteurs du même village ayant décidé de se regrouper pour améliorer les conditions de transport et la proximité circulatoire de leurs produits. Les entreprises de la classe 3 combinent, quant à elles, les proximités géographique et circulatoire. Dans cette classe, ces entreprises très exportatrices mobilisent également la proximité organisée pour développer des partenariats de longue durée avec les importateurs : la proximité organisée supplante dans ce cas la proximité géographique. Enfin, les entreprises de la classe 4 s'appuient en premier lieu sur la proximité géographique. Pour l'ensemble de nos entreprises, les proximités organisées, circulatoire et géographique, sont ainsi intrinsèquement liées et jouent chacune, selon les moments et selon les acteurs, un rôle moteur dans la constitution des partenariats.

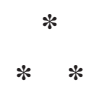

Les acteurs de la filière des vins de Bourgogne prennent peu à peu conscience de l'importance de la logistique aval. Pour répondre aux nouveaux enjeux, certains transporteurs ou logisticiens choisissent de développer des outils informatiques communs et des services (possibilité de suivre les expéditions sur le site web du transporteur, fourniture d'étiquettes autocollantes préimprimées, etc.) afin de construire une proximité avec les entreprises de la filière et disposer ainsi d'avantages compétitifs par rapport à leurs concurrents (Fulconis, Paché 2011). Néanmoins, seule une minorité d'entreprises vitivinicoles possède aujourd'hui une vision globale de la logistique aval, intégrant d'autres fonctions que le seul transport comme le stockage ou la préparation des commandes. Elles gèrent leur logistique dans le cadre d'un groupe, d'une Union de coopératives ou d'une structure collective. Une proportion importante d'entreprises n'envisage pas par contre une mutualisation de leur logistique. Cette réticence est plus marquée chez les viticulteurs qui ne veulent pas partager des informations sur les marchés et divulguer le nom de leurs clients à d'autres viticulteurs.

Au-delà de la question de la combinaison des proximités en jeu, un certain nombre de questions méritent d'être prolongées : la manière dont se construisent ces relations entre entreprises et prestataires logisticiens et dont ces derniers gèrent « les aspects confidentialité », primordiaux dans la filière vin ; ou encore l'éventuel risque d'enfermement dans des systèmes de relations qui s'appuient sur des codes communs de communication mais aussi sur des systèmes informatiques intégrés. De même, nous avons montré que l'utilisation de la notion de proximité circulatoire permet de mieux appréhender la prise en compte des transports de biens entre les entreprises. La proximité 
circulatoire est positivement reliée au type de transport majoritairement utilisé (fret ou messagerie), à la capacité à se regrouper et à concentrer les envois mais aussi à élaborer une véritable stratégie logistique avec la mise en place d'indicateurs de suivi (délais, taux de casse ou de vol, degré de satisfaction des clients, maîtrise des coûts), $\mathrm{du}$ recrutement d'un responsable logistique et/ou du recours aux logisticiens pour un certain nombre de services. Cependant, notre méthodologie ne nous permet que de capter partiellement cette proximité entre l'entreprise et le prestataire, et non sur l'ensemble de la chaîne logistique.

Si le cadre théorique proposé par les auteurs de la proximité permet de considérer de manière renouvelée les questions de coordination et stratégies d'acteurs, les travaux proposant des études empiriques et des indicateurs des différentes proximités méritent d'être développés. Nous avions l'objectif de proposer une telle analyse pour le secteur vitivinicole et si ces résultats restent exploratoires, ils ont néanmoins le mérite de nous aider à mieux appréhender la complexité des phénomènes à l'œuvre.

Nous tenons à remercier le Conseil régional de Bourgogne et le Bureau interprofessionnel des Vins de Bourgogne ainsi que le CESAER et Agrosup Dijon pour avoir financé un postdoctorant durant un an pour réaliser ce travail. Nous remercions également Tarek Ben Hassen qui a collecté les données auprès des différents acteurs de la filière vin en Bourgogne.

\section{RÉFÉRENCES BIBLIOGRAPHIQUES}

Adamo C. (2004). A global perspective of the wine supply chain-the case of Argentinean wineries and the U.S. market. Mémoire de Master. Massachusetts Institute of Technology.

Bellet M., Kirat T., Largeron C. (dir.) (1998). Approches multiformes de la proximité. Paris, Hermès.

Boissinot A., Kacioui-Maurin E. (2009). L'innovation envisagée comme une stratégie « d'enracinement » dans le canal de distribution par le prestataire de services logistiques. Logistique et Management, vol. 17, $\mathrm{n}^{\circ} 2$, pp. 7-16.

Boschma R. (2004). Proximité et innovation. Économie rurale, $\mathrm{n}^{\circ}$ 280, mars-avril, pp. 8-24.

Bouba-Olga O., Carrincazeaux C., Coris C. (2008). La proximité, 15 ans déjà ! Première partie : propositions théoriques. Revue d'Économie Régionale et Urbaine, $\mathrm{n}^{\circ} 3$, pp. 279-287.

Brulhart F. (2002). Le rôle de la confiance dans le succès des partenariats verticaux logistiques : le cas des coopérations entre industriels agroalimentaires et prestataires logistiques. Revue Finance Contrôle Stratégie, vol. 5, n 4 , pp. 51-77.

Burmeister A., Colletis-Wahl K. (1997). Les interactions production-transport-espace : Quelle(s) logique(s) de proximité(s) ? Revue d'Économie Régionale et Urbaine, $\mathrm{n}^{\circ} 3$, pp. 363-386.

Burmeister A. (2000). Familles logistiques Propositions pour une typologie des produits transportés pour analyser les évolutions en matière d'organisation des transports et de la logistique. Convention DRAST, $\mathrm{n}^{\circ} 98 \mathrm{MT}$ 87, octobre 2000, 126 p.

Burmeister A., Djellal F. (2004). L'impact des technologies de l'information sur l'organisation spatiale des activités de services : le cas du transport routier de marchandises et des services intensifs en connaissance. Innovations, vol. 1, n 19 , pp. 53-73.

Colin J. (2005). Le supply chain management existe-t-il réellement ? Revue française de gestion, vol. 3, n 156, pp. 135-149. 
Dornier P., Fender M. (2012). La logistique globale et le supply chain management. Paris, Eyrolles-Éditions d'Organisation.

El Ourdighi F., De Giovanni P., Tarondeau J.-C. (2008). L'expérience française du supply chain management. Revue Française de Gestion, vol. 6, n 186, pp. 89-116.

France Agrimer (2014). Grande étude nationale. Cafés, hôtels, restaurants. $116 \mathrm{p}$.

Fassio G. (2009). Normes environnementales et pratiques entrepreneuriales dans les secteurs de la logistique et des transports. Logistique et Management, vol. 17, $\mathrm{n}^{\circ}$ 1, pp. 5-18.

Filippi M., Torre A. (2003). Local organisations and institutions. How can geographical proximity be activated by collective projects ? International Journal of Technology Management, vol. 26, n 3-4, pp. 386-400.

Frigant V. (1996). Les espaces du juste-àtemps : une approche en termes de proximités. Revue d'Économie Régionale et Urbaine, $\mathrm{n}^{\circ}$ 4, pp. 777-794.

Fulconis F., Paché G. (2011). Entre innovation et optimisation : la décision en logistique à la croisée des chemins. Management \& Avenir, $\mathrm{n}^{\circ} 48 / 8$, pp. $158-178$. DOI : $10.3917 /$ mav.048.0158

Gilly J. P., Pecqueur B. (2000). Régulation des territoires et dynamiques institutionnelles de proximité : le cas de Toulouse et des Baronnies. In J.-P. Gilly, A. Torre (dir.), Dynamiques de proximité. Paris, L'Harmattan, pp. 131-164.

Giuliani E., Morrison A., Rabellotti R. (dir.) (2011). Innovation and technological catchup - The changing geography of wine production. Cheltenham, Edward Elgar, 220 p.

Gozé-Bardin I. (2009). Les défis de la logistique de distribution à l'horizon 2035 . Revue Management et Avenir, vol. 4, $\mathrm{n}^{\circ} 24$, pp. 217-236.

Jetin B., Lung Y. (2000). Un réexamen de la relation entre variété et échelle de production à partir de l'industrie automobile. Économie \& prévision, vol. $4, \mathrm{n}^{\circ} 145$, pp. 67-82.

Jouenne T., Masion J. (2007). La logistique dans les PME-PMI de l'agroalimentaire. Synthèse des résultats. Disponible en ligne : http://
www.supplychainmagazine.fr/TOUTEINFO/ETUDES/Enquete_logistique_agroalimentaire.pdf.

Kempainen K., Ari P. J. V. (2003). Trends in industrial supply chains and networks. International journal of physical distribution \& logistics management, vol. 33, pp. 701-719.

Masson S., Petiot V. (2013). Logistique et territoire, multiplicité des interactions et forces de régulation. Géographie, économie, société, vol. $15, \mathrm{n}^{\circ} 4$, pp. 385 à 412.

Markusen A. (2000). Des lieux-aimants dans un espace mouvant: une typologie des districts industriels. In G. Benko, A. Lipietz (dir.), $L a$ richesse des régions : la nouvelle géographie socio-économique, Paris, Presses universitaires de France, pp. 85-119.

May N. (1999). Districts et métropolisation : réflexions à partir de deux figures spatiales de réorganisations productives. In J.-M. Fontan, J.-L. Klein, D.-G. Tremblay (dir.), Entre la métropolisation et le village global. Québec, Presses de l'Université du Québec, pp. 27-47.

Michrafy M., Estampe D., Paul J. (2006). Les liens entre les indicateurs financiers, commerciaux et de la chaîne logistique : une analyse sectorielle européenne. Gestion, vol. 31, n 3, pp. 14-27.

OCDE (2005). Manuel d'Oslo, Principes directeurs pour le recueil et l'interprétation des données sur l'innovation. $3^{e}$ éd., 188 p.

Paché G., Sauvage T. (2004). La logistique, enjeux stratégiques. $3^{\mathrm{e}}$ édition, Paris, Vuibert.

Paché G. (2006). Approche spatialisée des chaînes logistiques étendues : de quelle(s) proximités(s) parle-t-on? Les Cahiers Scientifiques du Transport, $\mathrm{n}^{\circ}$ 49, pp. 9-28.

Pimor Y. (2003). Logistique : production, distribution, soutien, Paris, L'Usine nouvelle.

Poirel C., Bonet D. (2007). La chaîne logistique, un concept alternatif au canal de distribution pour étudier les relations entre clients et fournisseurs. In G. Paché, A. Spalanzani (dir.), La gestion des chaînes logistiques multi-acteurs : perspectives stratégiques. Grenoble, Presses universitaires de Grenoble, pp. 163-184. 
Porter M. E. (1985). Competitive Advantage: Creating and Sustaining Superior Performance. New York, Free Press.

Rallet A., Torre A. (2004). Proximité et localisation. Économie rurale, n ${ }^{\circ} 280$, pp. 25-41.

Reboud S., Mazzarol T., Soutar G. (2014). Low-tech vs high-tech entrepreneurship: a study in France and Australia. Journal of Innovation Economics \& Management, vol. $2, \mathrm{n}^{\circ} 14$, pp. 121-141.

Réjalot M. (2004). La logistique vinicole : Un nouvel acteur dans la filière vin de bordeaux. Sud-Ouest européen, $\mathrm{n}^{\circ} 18$, pp. 119-129.

Rivière-Giordano G. (2012). Structuration de l'organisation logistique des entreprises agroalimentaires du Languedoc-Rousillon. In M. Domergue, J.-P. Couderc, G. RivièreGiordano, C. Maurel (dir.), Dynamiques des entreprises agroalimentaires (EAA) du Languedoc-Rousillon 1998-2010. Série Recherches MOISA, décembre, pp. 113-125.

Shearmur R. (2011). Innovation, Regions and Proximity: From Neo-Regionalism to Spatial Analysis. Regional Studies, vol. 45, $\mathrm{n}^{\circ}$ 9, pp. 1125-1243.
Tanguy C., Martin M. (2015). Le développement durable dans la filière viticole bourguignonne : stratégies et pratiques. Innovations. Revue d'Économie et de Management de l'Innovation, $\mathrm{n}^{\circ}$ 46, pp. 141-160.

Torre A. (2008). On the role played by temporary geographical proximity in knowledge transmission. Regional Studies, vol. 42, $\mathrm{n}^{\circ}$ 6, pp. 869-899.

Torre A., Rallet A. (2005). Proximity and Localization. Regional Studies, vol. 39, $\mathrm{n}^{\circ} 1$, pp. 47-59.

Touzard J. M. (2008). La construction économique et politique des marchés : l'exemple de la reconversion viticole en LanguedocRoussillon. Les Cahiers du CEVIPOF, $\mathrm{n}^{\circ}$ 48, pp. 113-140.

Tremblay D. G., Klein J. L., Fontan J. M., Rousseau S. (2003). Proximité territoriale et innovation : une enquête sur la région de Montréal. Revue d'économie régionale et urbaine, vol. 5, pp. 835-852.

Zimmermann J. B. (2008). Le territoire dans l'analyse économique. Revue française de gestion, vol. 4, $\mathrm{n}^{\circ} 184$, pp. 105-118. 